\title{
Phosphoribosyl Pyrophosphate Amidotransferase Promotes the Progression of Thyroid Cancer via Regulating Pyruvate Kinase M2
}

This article was published in the following Dove Press journal: OncoTargets and Therapy

\author{
Bing Liu' ${ }^{1, *}$ \\ Meiyue Song ${ }^{2, *}$ \\ Huadong Qin' \\ Bin Zhang' \\ Yao Liu' \\ Yu Sun' \\ Yanfei Ma' \\ Tiefeng Shi $\mathbb{D}^{\prime}$
}

'The 4th Department of General Surgery, The Second Affiliated Hospital of Harbin Medical University, Harbin City I50086, Heilongjiang Province, People's Republic of China; ${ }^{2}$ The Pathology Department, The Second Affiliated Hospital of Harbin Medical University, Harbin City I50086, Heilongjiang Province, People's Republic of China

*These authors contributed equally to this work

\begin{abstract}
Background: Pyruvate kinase is an enzyme that catalyzes the conversion of phosphoenolpyruvate and ADP to pyruvate and ATP in glycolysis and plays a role in regulating cell metabolism. It is reported that the activity of pyruvate kinase is increased in cancers. Phosphoribosyl amidotransferase (PPAT) is reported to be a crucial regulator for pyruvate kinase activity in lung cancer. However, its role in thyroid cancer remains largely unknown. Materials and Methods: Immunohistochemical analysis and qRT-PCR were used to detect the expression of PPAT in thyroid cancer samples. Both gain-of-function and loss-of-function models were constructed in thyroid cancer cell lines and the biological functions of PPAT on cellular phenotypes were studied using CCK-8 assay and transwell assay in vitro, respectively. Then, Western blot was used to evaluate the change of PKM2 and downstream signal pathways after PPAT was overexpressed or knocked down.

Results: Immunohistochemical analysis showed increased expression of PPAT in thyroid cancer tissues, and it was associated with unfavorable pathological characteristics. Knockdown and overexpression assays suggested that altering PPAT expression modulated cell proliferation, migration, and invasion. In terms of mechanism, PPAT could positively regulate the expression of PKM2 and activate ERK and STAT3 signaling pathways.
\end{abstract}

Conclusion: PPAT plays crucial roles in regulating proliferation, migration, and invasion of thyroid cancer cells via activating PKM2, ERK, and STAT3.

Keywords: thyroid cancer, PPAT, PKM2, proliferation, migration, invasion

\section{Introduction}

Thyroid carcinoma (TC) is the most common endocrine malignancy in the world. Papillary thyroid carcinoma (PTC) is the main pathological type of TC, accounting for $80 \%$ of all TC cases. ${ }^{1,2}$ In recent decades, the incidence of TC has been increased gradually, mainly PTC. ${ }^{3}$ Although the prognosis of TC is relatively good, about $10 \%$ of cases are more invasive and deadly with high potential of distant metastasis. ${ }^{4,5}$ Therefore, in-depth exploration of the mechanism of TC occurrence and progression is still necessary.

Phosphoribosyl pyrophosphate amidotransferase (PPAT) is a key rate-limiting enzyme for purine metabolism. ${ }^{6,7}$ Studies show that changes in the enzyme activity caused by mutations in the PPAT gene lead to disorders in purine nucleotide metabolism, increasing the expression level of uric acid and causing diseases such as hyperuricemia and gout. ${ }^{8,9}$ In cancer biology, PPAT gene is mutated in some cases of gastric and colorectal cancers, which is related to the high microsatellite instability. ${ }^{10}$ Additionally,
Correspondence: Tiefeng Shi

The 4th Department of General Surgery, The Second Affiliated Hospital of Harbin Medical University, No. 246, Nangang

District, Harbin City 150086, Heilongjiang

Province, People's Republic of China

Tel +86 45I-866054I9

Email qinguyou9@163.com 
PPAT is confirmed to be highly expressed in lung adenocarcinoma tissues; overexpression of PPAT significantly promotes tumor cell proliferation and invasion via activating pyruvate kinase $(\mathrm{PK})$ and can function as a biomarker for aggressive lung adenocarcinoma. ${ }^{11}$ However, the expression and function of PPAT in TC and its mechanism have not yet been elucidated.

$\mathrm{PK}$ is a rate-limiting enzyme in the glycolytic pathway. It catalyzes the substrate phosphoenolpyruvate (PEP) to produce pyruvate and release energy. It can encode four isoenzymes, and among them, pyruvate kinase M2 (PKM2) is reported to be a key regulator of tumor cell metabolism, growth, and metastasis. ${ }^{12,13}$ With the up-regulation of PKM2, the enzyme structure changes from a traditional tetramer to a dimer, which has a weaker affinity for PEP, leading to changes in tumor cell metabolism, thus allowing tumor cells to proliferate with limited nutritional supply. ${ }^{14,15}$ Many studies show that PKM2 is increased in a variety of human cancers. PKM2 plays a crucial regulatory role in the carcinogenesis, proliferation, migration, and invasion of tumor cells. For example, in hepatocellular carcinoma, PKM2 interacts with the nuclear sterol regulatory element-binding protein 1a (SREBP-1 $\alpha$ ) to activate adipogenesis and promote cell proliferation; highly expressed PKM2 is significantly associated with poor prognosis of patients with liver cancer. ${ }^{16,17}$ PKM2 is significantly overexpressed in gallbladder cancer tissues, and up-regulation of PKM2 can promote tumorigenesis. ${ }^{18}$ It is also reported that PKM2 is involved in the progression of TC. ${ }^{19}$ Additionally, the activation or upregulation of PKM2 could activate multiple cancer-related pathways such as ERK signaling and STAT3 signaling. ${ }^{20-22}$ However, the expression of PKM2 in TC and its related mechanisms remain to be further explored.

The purpose of this study is to explore the expression and clinical significance of PPAT in TC, and further explore the effect of PPAT on the malignant phenotypes of TC cells and its related mechanisms. This study confirmed that PPAT was highly expressed in TC tissues and cells, and that patients with high expression of PPAT had unfavorable prognosis. In addition, PPAT was involved in promoting the proliferation and metastasis of TC cells by regulating PKM2 and activating ERK and STAT3 signaling pathways.

\section{Materials and Methods}

\section{Tissue Sample}

All participating patients signed informed consents prior to the study. This study was backed by Ethics Review Board of the Second Affiliated Hospital of Harbin Medical
University. Cancer tissues $(\mathrm{n}=50)$ and non-cancerous tissues $(\mathrm{n}=50)$ samples from May 2014 to May 2018 were randomly selected in this study. All samples were stored in liquid nitrogen at $-196^{\circ} \mathrm{C}$ for subsequent experiments.

\section{Immunohistochemistry (IHC)}

Streptomycin avidin-peroxidase (SP) method was used for IHC staining. The specimens were prepared into paraffin sections with a thickness of $4 \mu \mathrm{m}$. The sections were baked at $70^{\circ} \mathrm{C}$, and then paraffin sections were dehydrated with different gradients of ethanol, and then antigen repair was performed. After that, $\mathrm{H}_{2} \mathrm{O}_{2}$ solution was added in order to inactivate endogenous enzymes at room temperature for $10 \mathrm{~min}$ and washed with PBS for 10 min. After that, anti-PPAT antibody (1: 100, MA525,978, Invitrogen, Carlsbad, CA, USA) and anti-PKM2 antibody (1: 200, ab137852, Abcam, Shanghai, China) were added and the sections were incubated overnight at $4^{\circ} \mathrm{C}$. Then the sections were rinsed with PBS for 3 times, and the secondary antibody was added to incubate the section for $30 \mathrm{~min}$. After rinsing with PBS for 3 times, the sections were stained with DAB. Ultimately, the sections were observed and scored by two independent pathologists.

\section{Cell Culture}

Human TC cell lines (K1, KTC-1, BHP5-16, and BHP2-7 cells) and human thyroid follicular epithelial cell lines (Nthy-ori 3-1) were purchased from Shanghai Institute of Biochemistry and Cell Biology, CAS (Shanghai, China). All the above cells were cultured in Dulbecco's Modified Eagle's Medium (DMEM) (HyClone, Logan, UT, USA) containing $10 \%$ fetal bovine serum (FBS) (Invitrogen, Carlsbad, CA, USA) in an incubator with $5 \%$ volume fraction of $\mathrm{CO}_{2}$ at $37^{\circ} \mathrm{C}$. The medium was changed every 2-3 days, and when the cell grew to 80-90\% confluence, $0.25 \%$ trypsin (Roche, Basel, Switzerland) was used for subculture.

\section{Cell Transfection}

BHP5-16 and KTC-1 cells were inoculated into a $60 \mathrm{~mm}$ culture plate at a density of $1 \times 10^{6} / \mathrm{mL}$, and cultured for $24 \mathrm{~h}$, and then transfected. PPAT overexpression plasmid, PPAT siRNA, PKM2 overexpression plasmid, PKM2 siRNA, and the corresponding negative controls were purchased from RiboBio Co, Ltd. (Guangzhou, China). Transfection was performed with lipofectamine ${ }^{\mathrm{TM}} 2000$ (Invitrogen, Carlsbad, 
USA) according to the instructions. After $24 \mathrm{~h}$ of transfection, Western blot was used to detect transfection efficiency.

\section{RNA Extraction and Quantitative Real-Time Polymerase Chain Reaction (qRT-PCR)}

According to the manufacturer's instructions, total RNA was extracted from TC tissues and cells using TRIzol (Invitrogen, Shanghai, China), and cDNA was prepared using the FastQuan RT kit (Tiangen Biotech., Beijing, China). qRT-PCR analysis was performed using SYBR Premix Ex Taq ${ }^{\text {TM }}$ Kit (Takara Biotechnology Co., Ltd., Dalian, China) according to the manufacturer's instructions. Quantitative data were normalized to $\beta$-actin and the relative expression was calculated using the $2^{-\Delta \Delta \mathrm{Ct}}$ method. Each experiment was repeated three times. The specific primer sequence could be found in Table 1.

\section{Western Blot}

The total protein of TC cells was extracted with RIPA lysate (Beyotime, Shanghai, China), and the protein samples were boiled for $5 \mathrm{~min}$ for denaturation. The total protein was then prepared by SDS-PAGE. The protein was then transferred to PVDF membrane (Millipore, Bedford, MA, USA), which was washed with TBST, and blocked by $3 \%$ bovine serum albumin (BSA). After that, primary antibodies including anti-PPAT antibody (1: 1000, MA5-25,978, Invitrogen, Carlsbad, CA, USA), anti-PKM2 antibody (1: 1000, ab137852, Abcam, Shanghai, China), and anti- $\beta$-actin antibody (1: 2000, ab8826, Abcam, Shanghai, China) were loaded to incubate the membranes at $4^{\circ} \mathrm{C}$ for $8 \mathrm{~h}$. After that, the membrane was washed 3 times with TBST for 10 min each time. Subsequently, HRP-labeled secondary antibody (ab205718, 1:2000, Abcam, Shanghai, China) was loaded and the PVDF membrane was incubated for $2 \mathrm{~h}$. After that, ECL chemiluminescence kit (Millipore, Bedford, MA, USA) was used for showing the bands.

Table I Sequences Used for qRT-PCR

\begin{tabular}{|l|l|}
\hline PPAT & $\begin{array}{l}\text { F: GCGATTGAAGCACCTGTGGATG } \\
\text { R: CGGTTTTTACACAGCACCTCCAC }\end{array}$ \\
\hline PKM2 & $\begin{array}{l}\text { F: CCATCCTCTACCGHCCCCGTTG } \\
\text { R: CCAHCCCACAGGATGTTCTCGTC }\end{array}$ \\
\hline$\beta$-actin & $\begin{array}{l}\text { F: CCTTCCTGGGCATGGAGTCCT } \\
\text { R: GGAGCAATGATCTTGATCTTC }\end{array}$ \\
\hline
\end{tabular}

Abbreviations: F, forward; R, reverse; RT, reverse transcription.

\section{CCK-8 Assay}

BHP5-16 and KTC-1 cells were prepared as a single cell suspension, and the cell concentration was modulated to 2 $\times 10^{4} / \mathrm{mL}$, and inoculated into a 96-well plate with a volume of $100 \mu \mathrm{L} / w e l l$. At 24, 48, 72, and $96 \mathrm{~h}$ after inoculation, $10 \mu \mathrm{L} \quad \mathrm{CCK}-8$ reagent (Beyotime Biotechnology, Shanghai, China) was added into each well. After incubating for $1 \mathrm{~h}$, the absorbance of the wells at $450 \mathrm{~nm}$ was measured using a Bio-Rad mark microplate reader (Bio-Rad Laboratories, Shanghai, China).

\section{Transwell Experiment}

In migration assay, BHP5-16 and KTC-1 cells were harvested and resuspended with serum-free medium, and cell density was modulated to $5 \times 10^{5}$ cells $/ \mathrm{mL}$. $200 \mu \mathrm{L}$ cell suspension was added into the upper compartment of each transwell chamber $(8 \mu \mathrm{m}$ pore diameter, Corning, Shanghai, China); at the same time, $600 \mu \mathrm{L}$ medium containing 10\% FBS was dripped into the lower compartment. After the cells were cultured for $48 \mathrm{~h}$, the chamber was taken out from the incubator. The non-migrated cells on the membrane were wiped off with a cotton swab, and the migrated cells were fixed with anhydrous alcohol for 15 min and stained with $0.1 \%$ crystal violet solution for 20 min. After the membrane was washed by water gently and dried, five visual fields were randomly selected under a microscope, and the average number of migrated cells was calculated to evaluate the migration ability of the cells. In invasion assay, before the cells were inoculated, the Matrigel was used to cover the membrane of the transwell chamber.

\section{Statistical Method}

Analysis was performed using SPSS 17.0 statistical software (SPSS Inc., Chicago, IL, USA). The measurement data were expressed as mean \pm standard deviation $(\mathrm{x} \pm \mathrm{s})$. Comparisons between two groups were tested by $t$-test. The counting data were expressed in a fourfold table, and the difference between the two groups was analyzed by $\chi^{2}$ test. The difference was statistically significant with $P<0.05$.

\section{Results \\ PPAT mRNA and Protein Were Highly Expressed in TC Tissues and Cells}

Firstly, we used IHC to detect the expression of PPAT protein in 50 cases of TC tissue samples and 50 cases of 
normal thyroid tissues. The strong positive rate of PPAT protein in TC tissue samples was $46 \%$, the weak positive rate was $22 \%$, and the rate was $32 \%$. Compared with normal tissues, the expression of PPAT protein in TC tissues was significantly up-regulated $(P<0.05$, Figure 1A). Subsequently, the expression of PPAT mRNA in TC tissues was detected by qRT-PCR. It was found that PPAT mRNA was remarkably upregulated in TC tissues compared to normal tissues $(P<0.05$, Figure 1B). Additionally, compared with normal thyroid follicular epithelial cell Nthy-ori 3-1, PPAT mRNA expression was up-regulated in all of the TC cells $(P<0.05$, Figure 1C). Next, we used Western blot to detect the expression of PPAT protein in TC cells. Consistently, compared with Nthy-ori 3-1 cell, the expression of PPAT protein in TC cell lines was markedly increased $(P<0.05$, Figure 1D). Additionally, to explore the relationship between PPAT expression and prognosis of TC patients, we used the GEPIA database to analyze the correlation between PPAT mRNA expression and prognosis of TC patients. The results implied that the overall survival time of TC patients with high expression of PPAT was significantly shorter than that of patients with low expression of PPAT $(P=0.002$, Figure $1 \mathrm{E})$. The above results demonstrated that PPAT probably exerted a carcinogenic role in the progression of $\mathrm{TC}$ and could function as a potential marker of poor prognosis in TC patients.

\section{Correlation Between PPAT Expression and Clinicopathological Parameters in TC Patients}

Subsequently, we used chi-square test to analyze the correlation between PPAT expression and the pathological parameters of TC patients (Table 2). The results signified that the high expression of PPAT in TC tissues was significantly related to the larger tumor size $(P=$ $0.0227)$, positive lymph node metastasis $(P=0.0184)$, and increased TNM stage $(P=0.0087)$, and it was not conspicuously related to gender, age, nodular goiter, and unilateral or bilateral thyroid $(P>0.05)$. These data further implied that PPAT was involved in the progression of TC.
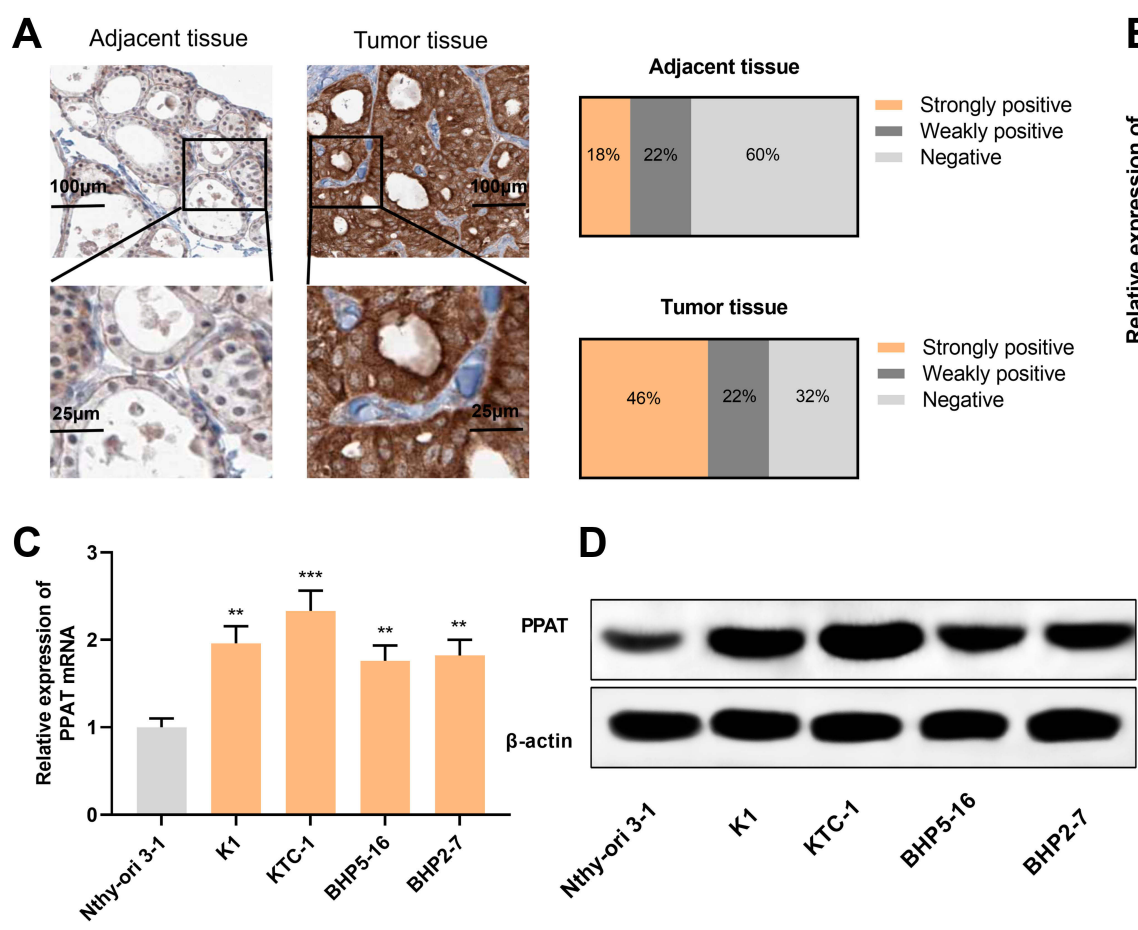

B
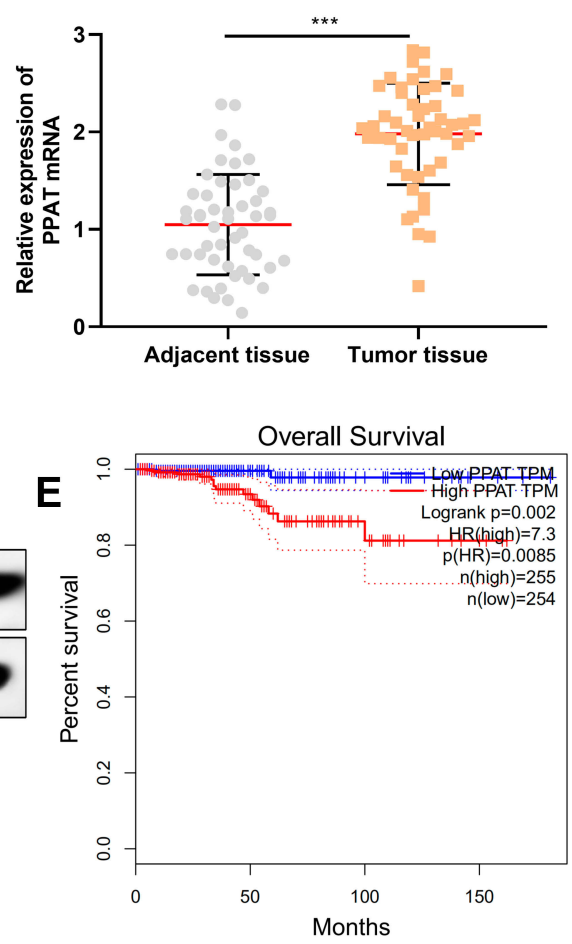

Figure I PPAT was highly expressed in TC tissues and cells. (A) We used IHC to detect the expression of PPAT protein in tissue samples from 50 TC patients and 50 normal thyroid tissues. (B) qRT-PCR was used to detect the expression of PPAT mRNA in TC tissues and normal thyroid tissues. (C) qRT-PCR was used to detect PPAT mRNA expression in normal thyroid follicular epithelial cells and TC cells. (D) Western blot was used to detect PPAT protein expression in normal thyroid follicular epithelial cells and TC cells. (E) The relationship between the expression of PPAT and the prognosis of TC patients was analyzed by GEPIA database. The experimental results were analyzed by Student's $t$-test, and the difference was statistically significant with $P<0.05$. $* * P<0.01$ and $* * * P<0.001$. 


\section{PPAT Promoted TC Cell Proliferation and Metastasis}

Then, we continued to explore the biological functions of PPAT in TC cells. We selected BHP5-16 cells with the lowest PPAT expression and KTC-1 cells with the highest PPAT expression in TC cells to construct PPAT overexpression and knockdown models, respectively. Compared with the NC group, PPAT expression was significantly upregulated after overexpression of PPAT in BHP5-16 cells for 24,48 , and $72 \mathrm{~h}$. Compared with the si-NC group, the expression of PPAT in KTC-1 cells was significantly down-regulated at 24,48 , and $72 \mathrm{~h}$ after transfection with PPAT siRNAs. $(P<0.05$, Figure 2A, Supplementary Figure 1A-D). Then, TC cell proliferation and metastasis were detected by CCK-8 and transwell assays, respectively. The results indicated that compared with the NC group, overexpression of PPAT considerably

Table 2 Correlation Between PPAT Expression and Clinical Features of TC Patients $(\mathrm{N}=50)$

\begin{tabular}{|c|c|c|c|c|c|}
\hline \multirow[t]{2}{*}{ Characteristics } & \multirow{2}{*}{$\begin{array}{l}\text { Number } \\
\text { of } \\
\text { Patients }\end{array}$} & \multicolumn{2}{|c|}{$\begin{array}{l}\text { PPAT } \\
\text { Expression }\end{array}$} & \multirow[t]{2}{*}{$\chi^{2}$} & \multirow[t]{2}{*}{$P$ value } \\
\hline & & Low & High & & \\
\hline \multicolumn{6}{|l|}{ Gender } \\
\hline Male & 30 & 18 & 12 & 3.0000 & 0.0833 \\
\hline Female & 20 & 7 & 13 & & \\
\hline \multicolumn{6}{|l|}{ Age (years) } \\
\hline$\geq 45$ & 24 & 14 & 10 & 1.2821 & 0.2575 \\
\hline$<45$ & 26 & $\mathrm{II}$ & 15 & & \\
\hline \multicolumn{6}{|l|}{ Tumor size $(\mathrm{cm})$} \\
\hline$\leq 1$ & 28 & 10 & 18 & 5.1948 & 0.0227 \\
\hline$>1$ & 22 & 15 & 7 & & \\
\hline \multicolumn{6}{|l|}{ Nodular goiter } \\
\hline Negative & 29 & 16 & 13 & 0.7389 & 0.3900 \\
\hline Positive & 21 & 9 & 12 & & \\
\hline \multicolumn{6}{|l|}{$\begin{array}{l}\text { Lymph node } \\
\text { metastasis }\end{array}$} \\
\hline Negative & 18 & 13 & 5 & 5.5556 & 0.0184 \\
\hline Positive & 32 & 12 & 20 & & \\
\hline \multicolumn{6}{|l|}{$\begin{array}{l}\text { Unilateral or } \\
\text { bilateral }\end{array}$} \\
\hline Unilateral & 21 & 13 & 8 & $1.423 \mathrm{I}$ & 0.2329 \\
\hline Bilateral & 29 & 13 & 16 & & \\
\hline \multicolumn{6}{|l|}{ TNM stage } \\
\hline I + II & 19 & 14 & 5 & $6.876 I$ & 0.0087 \\
\hline III + IV & 31 & 11 & 20 & & \\
\hline
\end{tabular}

promoted the proliferation, migration, and invasion of BHP5-16 cells; compared with the control group, knockdown of PPAT significantly inhibited KTC-1 cell proliferation, migration, and invasion $(P<0.05$, Figure $2 \mathrm{~B}-\mathrm{D})$. These results indicated that PPAT was involved in promoting the proliferation, migration, and invasion of TC cells.

\section{PKM2 mRNA and Protein Expressions Were Up-Regulated in TC Tissues and Cells}

Next, we investigated the expression of PKM2 in TC tissues with GEPIA database, and the results connoted that PKM2 expression was significantly up-regulated in TC tissues compared to normal tissues (Figure 3A). Next, we used immunohistochemistry to detect the expression of PKM2 protein in tissue samples from TC patients. As is shown, the strong positive rate of PKM2 protein in TC tissue samples was $52 \%$, the weak positive rate was $26 \%$, and the negative rate was $22 \%$. Compared with normal thyroid tissue tissues, the expression of PKM2 protein in TC tissues was significantly up-regulated $(P<$ 0.05, Figure 3B). Consistently, qRT-PCR results showed that compared with normal tissues and normal thyroid follicular epithelial cells, PKM2 mRNA was significantly overexpressed in TC tissues and cells $(P<0.05$, Figure $3 \mathrm{C}$ and $\mathrm{D})$. Besides, Western blot results manifested that compared with normal thyroid follicular epithelial cells, PKM2 protein was significantly enhanced in TC cells $(P<0.05$, Figure 3E). These results suggested that PKM2 was involved in promoting the progression of TC.

\section{PKM2 Participated in Promoting Malignant Progression of TC}

We also constructed cell lines with PKM2 overexpression and knockdown. Compared with the NC group, PKM2 expression was significantly up-regulated after overexpression of PKM2 in BHP5-16 cells for 24, 48, and 72 h. Compared with the si-NC group, the expression of PKM2 in KTC-1 cells was significantly down-regulated at 24,48 , and $72 \mathrm{~h}$ after transfection with PKM2 siRNAs. $(P<$ 0.05 , Figure 4A, Supplementary Figure $2 \mathrm{~A}-\mathrm{D})$. Cell proliferation assay was determined by CCK- 8 assay. The results signified that compared with the $\mathrm{NC}$ group, overexpression of PKM2 significantly promoted the proliferation of BHP516 cells, and knockdown of PKM2 significantly inhibited the proliferation of KTC-1 cells $(P<0.05$, Figure 4B). Moreover, through transwell experiments, it was also 

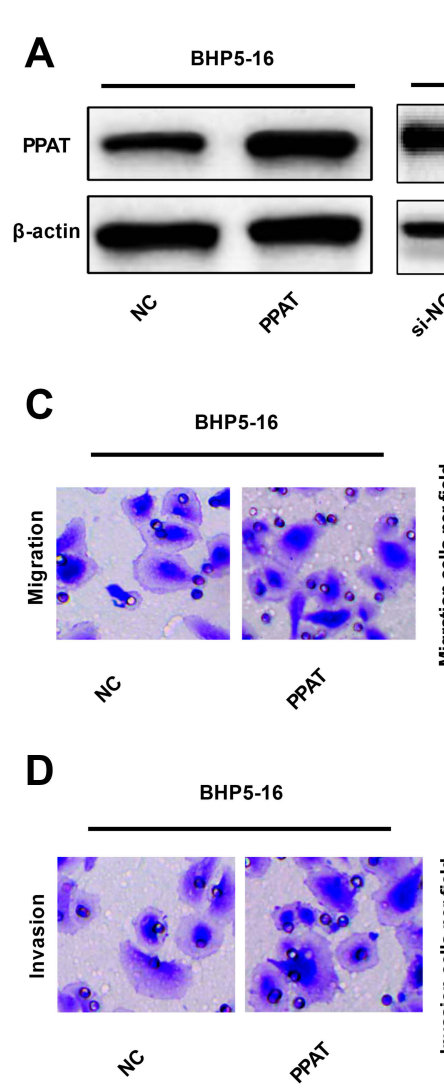
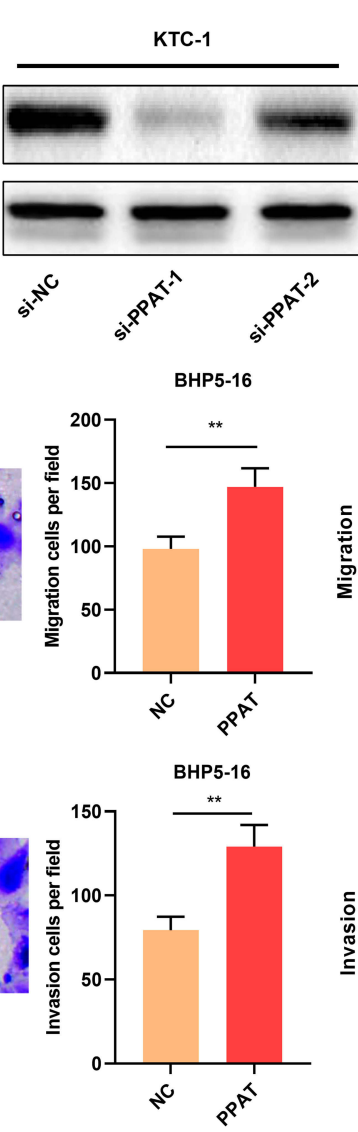

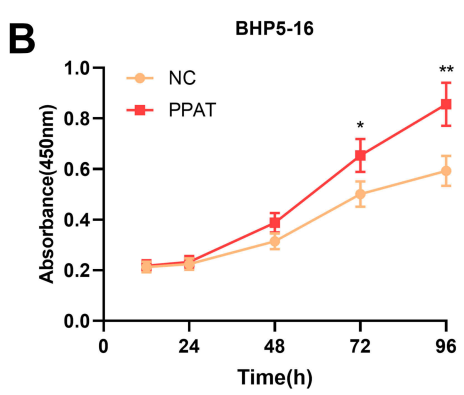

KTC-1

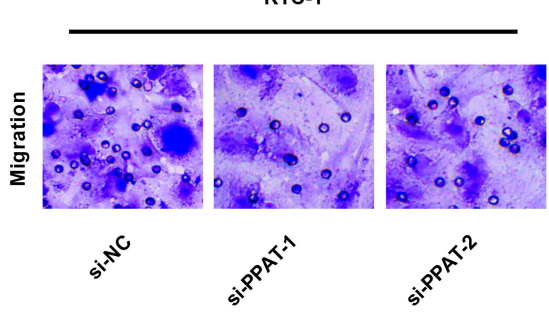

KTC-1

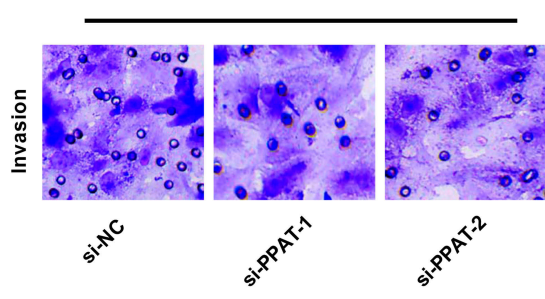

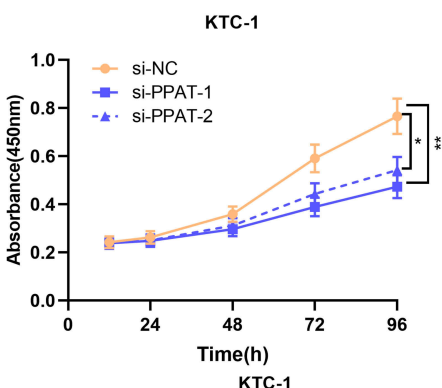
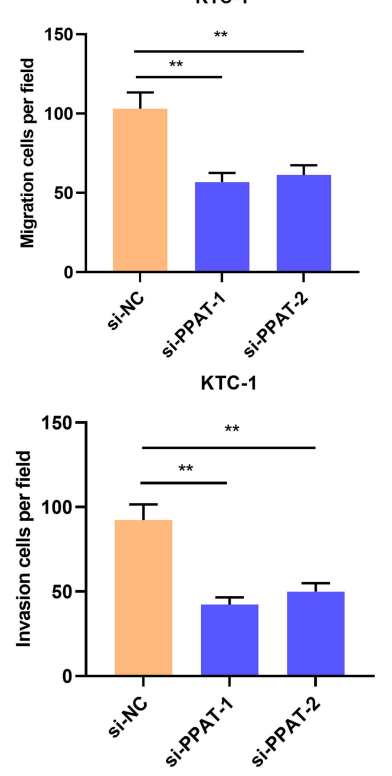

Figure 2 PPAT promoted TC cell proliferation, migration and invasion. (A) Western blot was used to detect the expression of PPAT protein in TC cells after overexpression or knockdown of PPAT. (B) CCK-8 method was used to detect the proliferation of BHP5-16 and KTC-I cells. (C and D) Transwell assay was used to detect the migration and invasion of BHP5-16 and KTC-I cells. The experimental results were analyzed by Student's $t$-test, and the difference was statistically significant with $P<0.05$. $* P<0.05$ and $* * P<0.01$.

confirmed that overexpression of PKM2 significantly promoted the migration and invasion of BHP5-16 cells, while knockdown of PKM2 played the opposite role $(P<0.05$, Figure $4 \mathrm{C}$ and D). The above results indicated that PKM2 promoted the proliferation, migration, and invasion of TC cells.

\section{PPAT Positively Regulated PKM2 Expression and Enhanced ERK/STAT3}

\section{Signaling Pathway}

Previous studies demonstrate that PPAT can regulate the activity of PKM2 and thus affect the progression of lung cancer cells. ${ }^{11}$ Inspired by this, we used qRT-PCR and Western blot to detect the expression of PKM2 in TC cells with PPAT overexpression and knockdown, respectively. The results connoted that PKM2 mRNA and protein expression were up-regulated in PPAT-overexpressing BHP5-16 and KTC-1 cells compared to the NC group. Compared to the si-
NC group, PKM2 mRNA and protein expression were decreased in BHP5-16 and KTC-1 cells with PPAT knockdown $(P<0.05$, Figure 5A-C, Supplementary Figure 3A- - ). Additionally, there was a positive correlation between the expressions of PPAT mRNA and PKM2 mRNA in TC tissue $(P<0.05$, Figure 5D). These data suggested that in TC cells, PPAT could positively regulate the expression of PKM2. Additionally, overexpression of PPAT significantly enhanced the expression levels of phosphorylated STAT3 (p-STAT3) and ERK (p-ERK), and knockdown of PKM2 reversed the effects of PPAT on STAT3 and ERK $(P<0.05$, Figure 5E). These results suggested that PPAT could promote TC progression via activating STAT3 and ERK signaling, which was mediated by PKM2.

\section{Discussion}

In cancer biology, aerobic glycolysis, also known as the Warburg effect, is a metabolic abnormality that often occurs during the development of tumors, ${ }^{23,24}$ This effect 

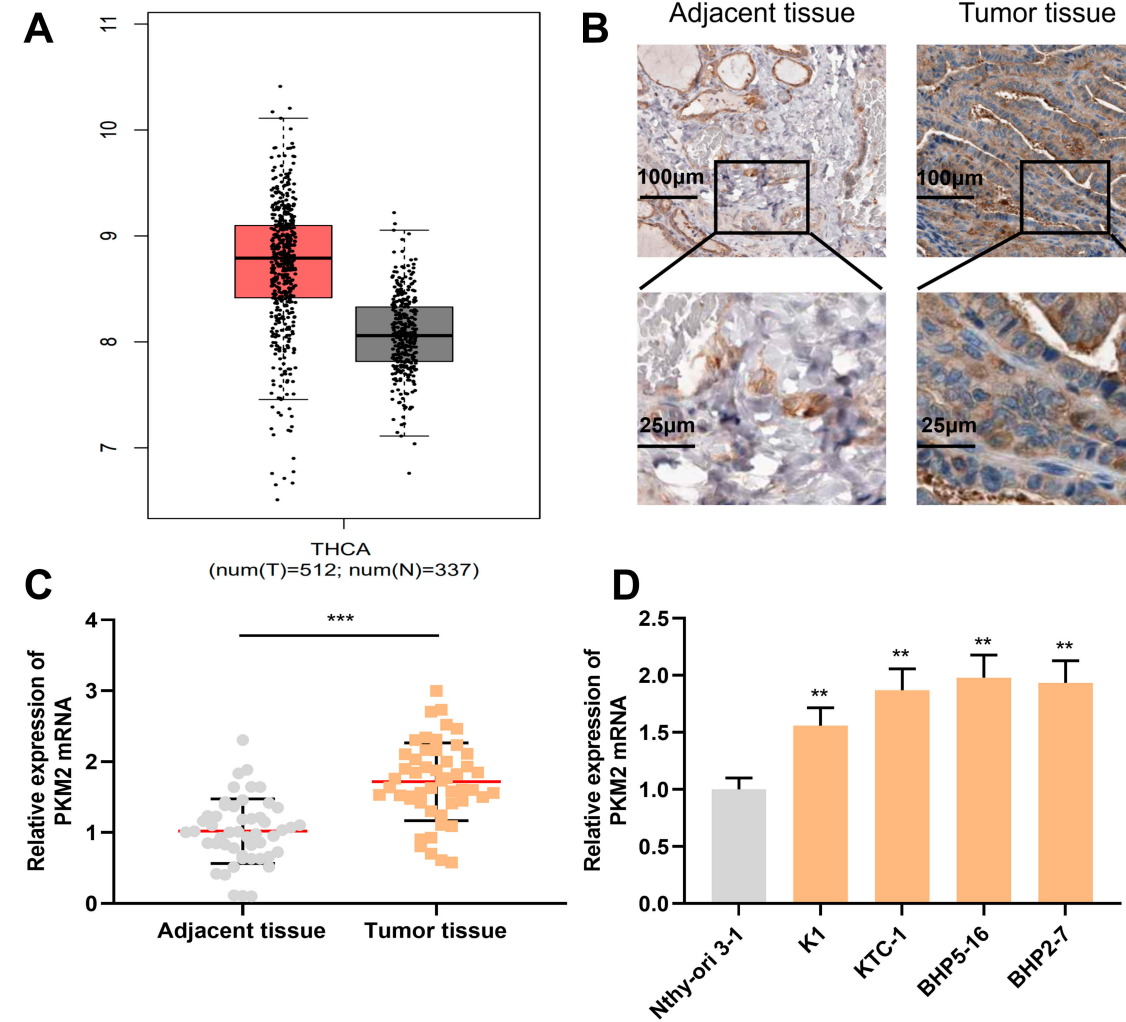
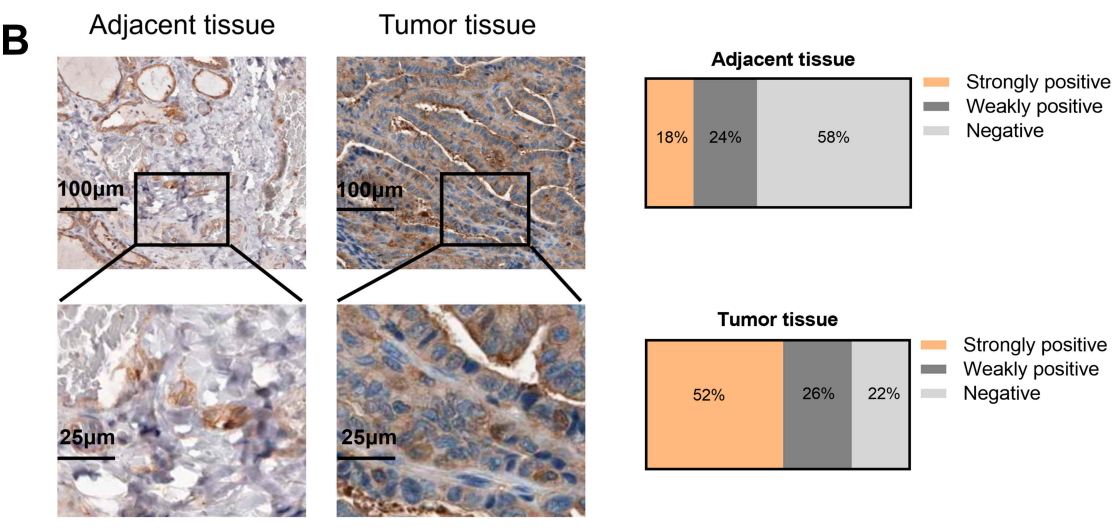

E
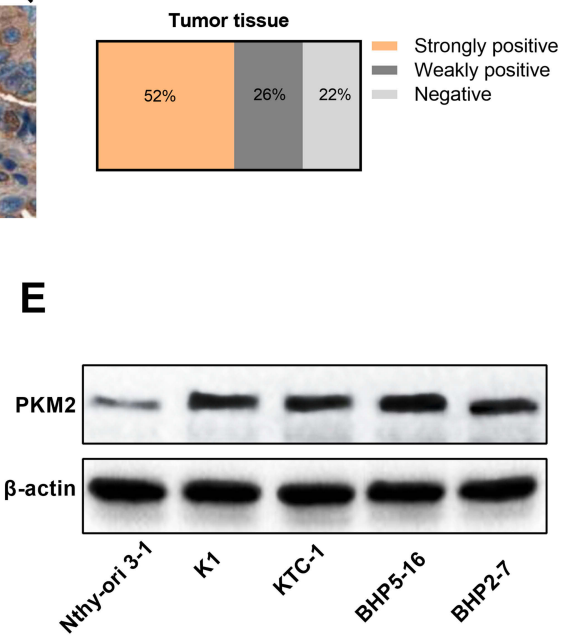

Figure 3 PKM2 expression was up-regulated in TC tissues and cells. (A) GEPIA database was used to analyze the expression of PKM2 in TC tissues. (B) Immunohistochemistry was used to detect the expression of PKM2 protein in tissue samples from TC patients and normal thyroid tissues. (C) qRT-PCR was used to detect the expression of PKM2 mRNA in TC tissues and normal thyroid tissues. (D) qRT-PCR was used to detect PKM2 mRNA expression in normal thyroid follicular epithelial cells and TC cells. (E) Western blot was used to detect PKM2 protein expression in normal thyroid follicular epithelial cells and TC cells. The experimental results were analyzed by Student's $t$-test, and the difference was statistically significant with $P<0.05$. $* * P<0.0 \mathrm{I}$ and $* * * P<0.00 \mathrm{I}$.

enables tumor cells to introduce glycolysis intermediate products into synthetic metabolic pathways, such as purine and pyrimidine biosynthesis, to ensure the sustaining proliferation of cancer cells. ${ }^{25}$ PPAT is a key regulator in the biosynthesis of purine. The protein encoded by PPAT gene is a member of the purine and pyrimidine phosphoribonucleotransferase family, which catalyzes the first step in the de novo biosynthesis of purine nucleotide. ${ }^{6,7}$ Previous studies show that PPAT can be a promising drug target for the treatment of lung cancer, leukemia, osteosarcoma, and other cancers. ${ }^{11,26-28}$ In this study, for the first time, we confirmed that PPAT expression was significantly upregulated in TC tissues and cells, and that patients with high expression of PPAT in tumor tissues had unfavorable pathological parameters. In addition, in vitro experiments confirmed that overexpression of PPAT could significantly promote the proliferation, migration, and invasion of TC cells, while knockdown had the opposite effect. The above results depicted that PPAT featured a cancer-promoting role in the malignant transformation of TC cells and was a potential therapy target for TC.
PKM2 is a key enzyme for glycolysis, which is commonly overexpressed in tumor cells. Enzymatic reaction catalyzed by it not only provides the necessary energy for tumor cell growth but also offers tumor cells with substrates for the synthesis of macromolecules. ${ }^{29,30}$ PKM2 expression is up-regulated in multiple cancers, and it can regulate many biological behaviors of cancer cells, such as cell proliferation, metastasis, and apoptosis. ${ }^{31-34}$ Some studies imply that the detection of PKM2 is of great value in the early diagnosis and prognosis prediction in lung cancer, breast cancer, gastrointestinal cancer, kidney cancer, and other tumors. ${ }^{35-37}$ It is also reported that PKM2 is significantly overexpressed in PTC, especially the cases harboring BRAF mutations, and its high expression is closely related to the advanced tumor stage and lymph node metastasis; additionally, PKM2 knockdown significantly inhibits PTC cell growth, lactic acid and ATP production, and glucose consumption. ${ }^{19}$ Our work confirmed that PKM2 was significantly overexpressed in TC tissues and cells. Overexpression of PKM2 significantly promoted the proliferation, migration, and invasion 

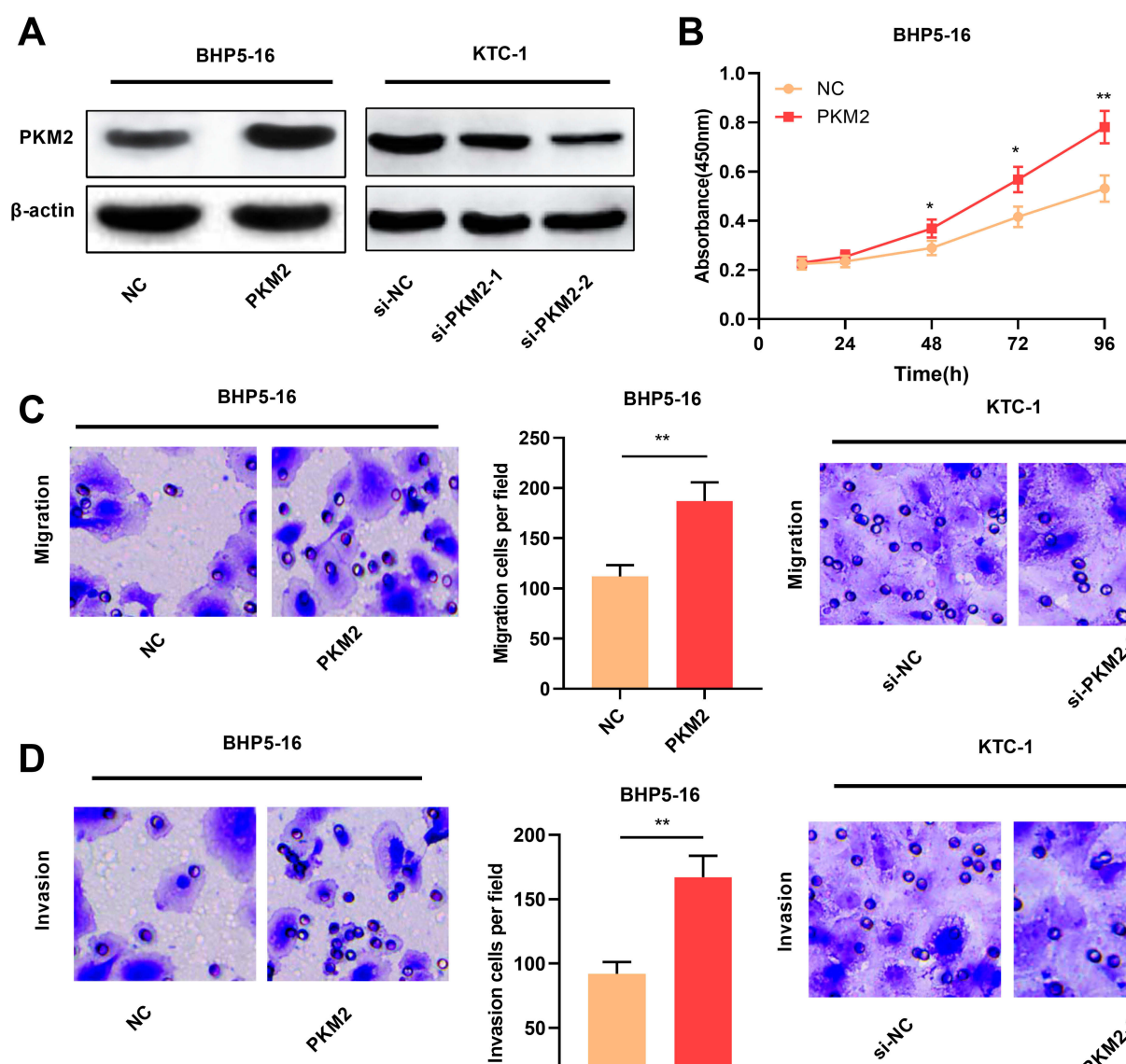
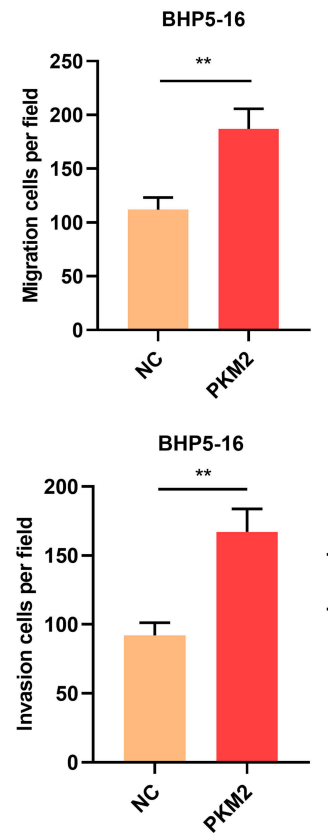

KTC-1

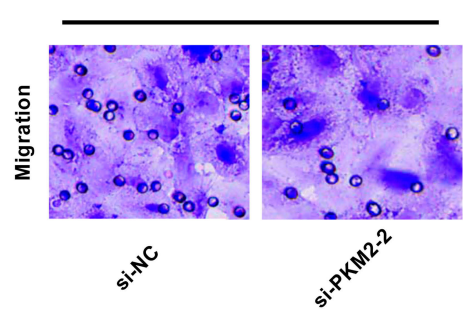

KTC-1
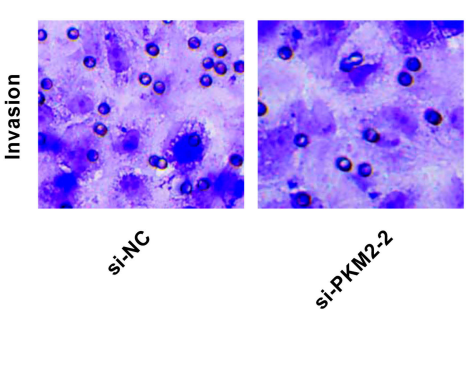

KTC-1

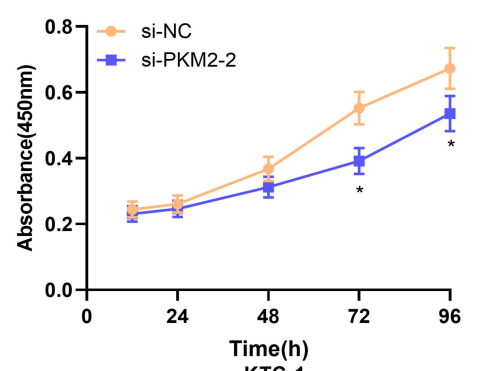

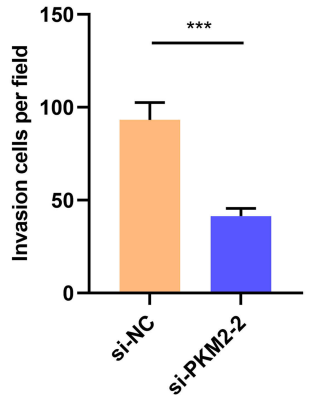

Figure 4 PKM2 promoted the proliferation, migration, and invasion of TC cells. (A) Western blot was used to detect the expression of PKM2 protein in TC cells after overexpression or knockdown of PKM2. (B) CCK-8 method was used to detect the proliferation of BHP5-16 and KTC-I cells after overexpressing or knocking down PKM2. (C and D) Transwell assay was used to detect BHP5-16 and KTC-I cell migration and invasion after overexpressing or knocking down PKM2. The experimental results were analyzed by Student's $t$-test, and the difference was statistically significant with $P<0.05$. $* P<0.05$, $* * P<0.01$, and $* * * P<0.001$.

of TC cells, while knockdown of PKM2 significantly reduced the malignant progression of TC cells. These demonstrations were consistent with previous reports. ${ }^{19}$

There are many factors contributing to the dysregulation of PKM2 in cancers, such as allosteric effects, transcriptional regulation, post-transcriptional modification, and protein interactions. ${ }^{38}$ Hypoxia-inducible factor, NF$\kappa \mathrm{B}$, and PPAR $\gamma$ have been verified to be involved in the transcriptional regulation of PKM2. ${ }^{39-41}$ Heat shock protein 90 regulates PKM2 activity through Thr-328 phosphorylation, thereby promoting glycolysis and proliferation, and inhibiting apoptosis of liver cancer cells. ${ }^{42}$ Up-regulation of PPAT expression in lung cancer can significantly enhance the activity of PKM2, thereby promoting tumor cell proliferation and invasion. ${ }^{11}$ In this work, through qRT-PCR and Western blot experiments, we confirmed that up-regulating PPAT expression could significantly enhance the expression of PKM2 mRNA and protein, while knockdown had the opposite effect, and the expression of PPAT mRNA and PKM2 mRNA in TC tissues were positively correlated. In addition, PPAT could activate ERK and STAT3 signaling pathways, which was mediated by PKM2. These data suggested that PPAT/ PKM2 was a crucial axis in the progression of TC.

In conclusion, this study confirms that PPAT is significantly overexpressed in TC tissues and cells, and its high expression is significantly related to the adverse clinicopathological characteristics of TC. Its knockdown or overexpression can significantly affect the biological behaviors of TC cells. In terms of mechanism, it is confirmed that PPAT can participate in promoting TC cell proliferation, migration, and invasion by regulating PKM2 expression and activating the ERK and STAT3 signaling pathways. In other words, PPAT is expected to 


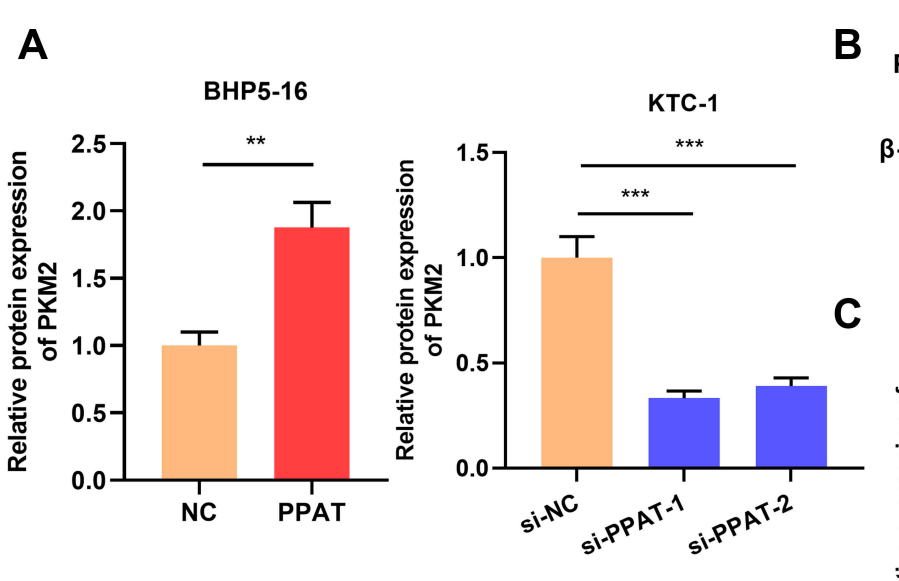

D

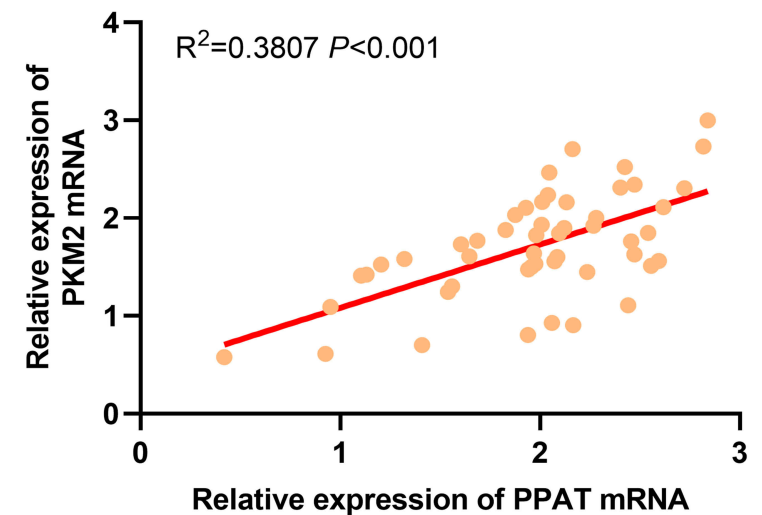

E
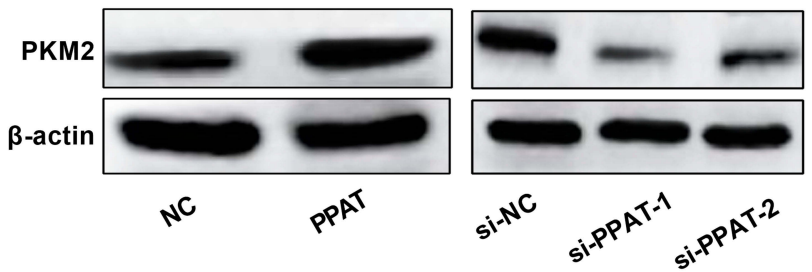

C
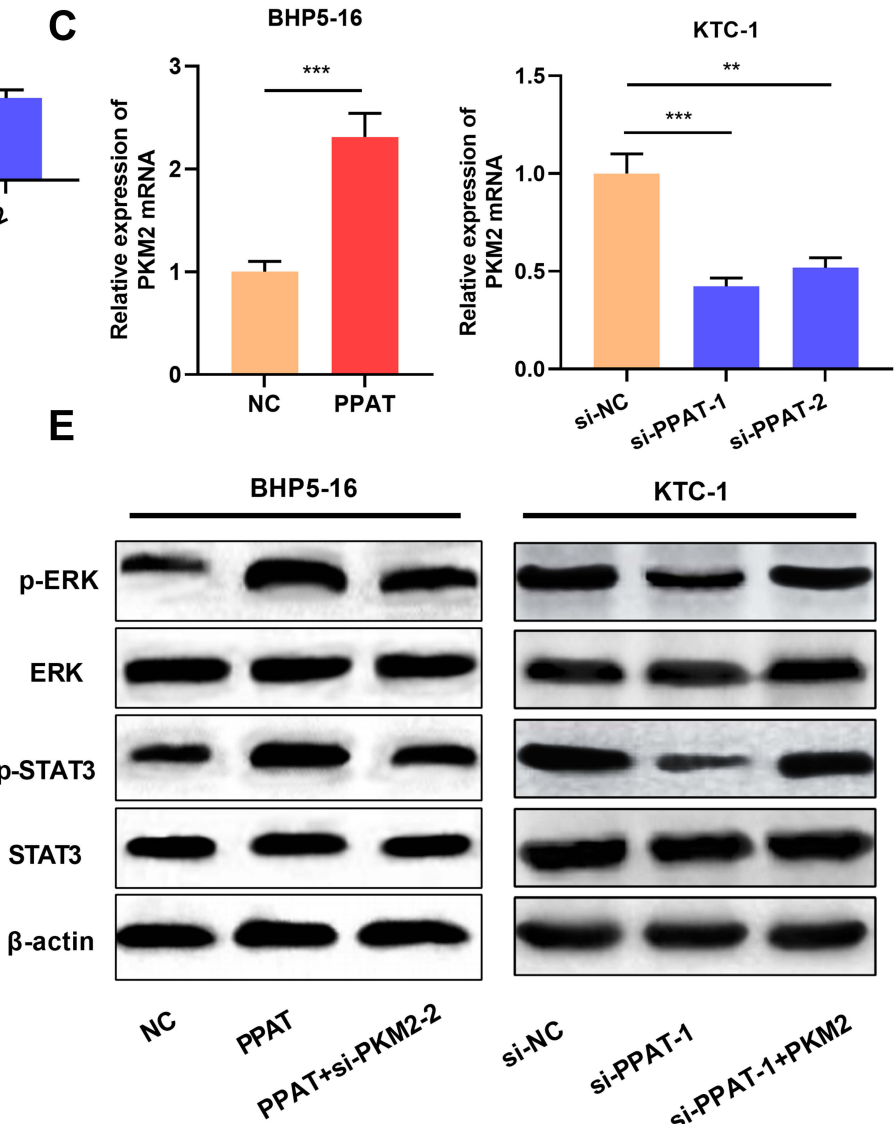

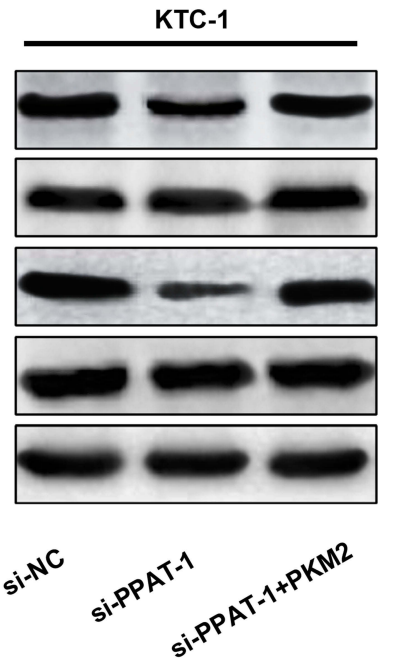

Figure 5 PPAT activated ERK and STAT3 signaling pathway by up-regulating PKM2 expression. (A and B) Western blot was used to detect the expression of PKM2 protein in BHP5-16 and KTC-I cells after overexpression or knockdown of PPAT. (C) qRT-PCR was used to detect the expression of PKM2 mRNA in BHP5-I6 and KTC-I cells after overexpression or knockdown of PPAT. (D) qRT-PCR was used to detect the correlation between PPAT mRNA and PKM2 mRNA expression in TC tissue. (E) Western blot was used to detect the p-ERK, ERK, P-STAT3, and STAT3 after PPAT and PKM2 were modulated in TC cells. The experimental results were analyzed by Student's $t$-test, and the difference was statistically significant with $P<0.05$. *** $P<0.01$ and $* * * P<0.00 \mathrm{I}$.

become a biomarker for $\mathrm{TC}$ diagnosis and a potential target for TC treatment. In the following studies, in vivo models are necessary to validate our demonstrations, and it is worth exploring the functions of PPAT/PKM2 axis on the metabolomics of TC cells to further clarify the mechanism by which PPAT/PKM2 axis promotes the malignant phenotypes of TC cells.

\section{Abbreviations}

PPAT, phosphoribosyl pyrophosphate amidotransferase; PKM2, pyruvate Kinase M2; TC, thyroid carcinoma; PTC, papillary thyroid carcinoma; PK, pyruvate kinase; PEP, phosphoenolpyruvate; SREBP-1a, sterol regulatory element-binding protein 1a; IHC, Immunohistochemistry; SP, streptomycin avidin-peroxidase; DMEM, Dulbecco's Modified Eagle's Medium; FBS, fetal bovine serum; qRTPCR, quantitative real-time polymerase chain reaction.

\section{Data Sharing Statement}

The data used to support the findings of this study are available from the corresponding author upon request.

\section{Ethics Statement}

Our study was approved by the Ethics Review Board of the Second Affiliated Hospital of Harbin Medical University. 


\section{Author Contributions}

All authors made substantial contributions to conception and design, acquisition of data, or analysis and interpretation of data; took part in drafting the article or revising it critically for important intellectual content; gave final approval of the version to be published; and agreed to be accountable for all aspects of the work.

\section{Disclosure}

The authors declare that they have no competing interests.

\section{References}

1. Zembska A, Jawiarczyk-Przybyłowska A, Wojtczak B, Bolanowski M. MicroRNA expression in the progression and aggressiveness of papillary thyroid carcinoma. Anticancer Res. 2019;39 (1):33-40. doi:10.21873/anticanres. 13077

2. Iglesias ML, Schmidt A, Ghuzlan AA, et al. Radiation exposure and thyroid cancer: a review. Arch Endocrinol Metab. 2017;61 (2):180-187. doi:10.1590/2359-3997000000257

3. Siegel RL, Miller KD, Jemal A. Cancer statistics, 2017. CA Cancer J Clin. 2017;67(1):7-30. doi:10.3322/caac.21387

4. Janjua N, Wreesmann VB. Aggressive differentiated thyroid cancer. Eur J Surg Oncol. 2018;44(3):367-377. doi:10.1016/j. ejso.2017.09.019

5. van der Tuin K, Ventayol Garcia M, Corver WE, et al. Targetable gene fusions identified in radioactive iodine refractory advanced thyroid carcinoma. Eur $J$ Endocrinol. 2019;180(4):235-241. doi:10.1530/EJE-18-0653

6. Woods RA, Roberts DG, Friedman T, Jolly D, Filpula D. Hypoxanthine: guanine phosphoribosyltransferase mutants in Saccharomyces cerevisiae. Mol Gen Genet. 1983;191(3):407-412. doi:10.1007/BF00425755

7. Smolina VS, Bekker ML. Properties of 5-phosphoryl-1-pyrophosphate amidotransferase from the yeast saccharomyces cerevisiae wild type and mutant with altered purine biosynthesis regulation. Biokhimiia. 1982;47(1):162-167.

8. Boban M, Kocic G, Radenkovic S, et al. Circulating purine compounds, uric acid, and xanthine oxidase/dehydrogenase relationship in essential hypertension and end stage renal disease. Ren Fail. 2014;36(4):613-618. doi:10.3109/0886022X.2014.882240

9. Hsu KC, Wang FS. Fuzzy optimization for detecting enzyme targets of human uric acid metabolism. Bioinformatics. 2013;29 (24):3191-3198. doi:10.1093/bioinformatics/btt564

10. Jo YS, Oh HR, Kim MS, Yoo NJ, Lee SH. Frameshift mutations of OGDH, PPAT and PCCA genes in gastric and colorectal cancers. Neoplasma. 2016;63(5):681-686. doi:10.4149/neo_2016_504

11. Goswami MT, Chen G, Chakravarthi BV, et al. Role and regulation of coordinately expressed de novo purine biosynthetic enzymes PPAT and PAICS in lung cancer. Oncotarget. 2015;6(27):23445-23461. doi:10.18632/oncotarget.4352

12. Jia L, Huang S, Yin X, Zan Y, Guo Y, Han L. Quercetin suppresses the mobility of breast cancer by suppressing glycolysis through Akt-mTOR pathway mediated autophagy induction. Life Sci. 2018;208:123-130. doi:10.1016/j.lfs.2018.07.027

13. Wan L, Xia T, Du Y, et al. Exosomes from activated hepatic stellate cells contain GLUT1 and PKM2: a role for exosomes in metabolic switch of liver nonparenchymal cells. FASEB J. 2019;33 (7):8530-8542. doi:10.1096/fj.201802675R

14. Dayton TL, Jacks T, Vander Heiden MG. PKM2, cancer metabolism, and the road ahead. EMBO Rep. 2016;17(12):1721-1730. doi:10.15252/embr.201643300
15. Li YH, Li XF, Liu JT, et al. PKM2, a potential target for regulating cancer. Gene. 2018;668:48-53. doi:10.1016/j.gene.2018.05.038

16. Zhao X, Zhao L, Yang H, et al. Pyruvate kinase M2 interacts with nuclear sterol regulatory element-binding protein 1a and thereby activates lipogenesis and cell proliferation in hepatocellular carcinoma. J Biol Chem. 2018;293(17):6623-6634. doi:10.1074/jbc. RA117.000100

17. Zhao R, Li L, Yang J, et al. Overexpression of pyruvate kinase M2 in tumor tissues is associated with poor prognosis in patients with hepatocellular carcinoma. Pathol Oncol Res. 2019:1-8.

18. Lu W, Cao Y, Zhang Y, et al. Up-regulation of PKM2 promote malignancy and related to adverse prognostic risk factor in human gallbladder cancer. Sci Rep. 2016;6:26351. doi:10.1038/srep26351

19. Feng C, Gao Y, Wang C, et al. Aberrant overexpression of pyruvate kinase M2 is associated with aggressive tumor features and the BRAF mutation in papillary thyroid cancer. $J$ Clin Endocrinol Metab. 2013;98(9):E1524-E1533. doi:10.1210/jc.2012-4258

20. Wang B, Liu S, Fan B, et al. PKM2 is involved in neuropathic pain by regulating ERK and STAT3 activation in rat spinal cord. $J$ Headache Pain. 2018;19(1):7. doi:10.1186/s10194-018-0836-4

21. Zheng B, Geng L, Zeng L, Liu F, Huang Q. AKT2 contributes to increase ovarian cancer cell migration and invasion through the AKT2-PKM2-STAT3/NF-кB axis. Cell Signal. 2018;45:122-131. doi:10.1016/j.cellsig.2018.01.021

22. Tang JC, Ren YG, Zhao J, Long F, Chen JY, Jiang Z. Shikonin enhances sensitization of gefitinib against wild-type EGFR non-small cell lung cancer via inhibition PKM2/stat3/cyclinD1 signal pathway. Life Sci. 2018;204:71-77. doi:10.1016/j.lfs.2018.05.012

23. Warburg O. On respiratory impairment in cancer cells. Science. 1956;124(3215):269-270.

24. Vander Heiden MG, Cantley LC, Thompson CB. Understanding the warburg effect: the metabolic requirements of cell proliferation. Science. 2009;324(5930):1029-1033. doi:10.1126/science.1160809

25. Vander Heiden MG, Lunt SY, Dayton TL, et al. Metabolic pathway alterations that support cell proliferation. Cold Spring Harb Symp Quant Biol. 2011;76:325-334. doi:10.1101/sqb.2012.76.010900

26. Bibi N, Parveen Z, Nawaz MS, Kamal MA. In silico structure modeling and molecular docking analysis of Phosphoribosyl Pyrophosphate Amidotransferase (PPAT) with antifolate inhibitors. Curr Cancer Drug Targets. 2019;19(5):408-416. doi:10.2174/ 1568009619666181127115015

27. Zhu Y, Li T, Ramos da Silva S, et al. A critical role of glutamine and asparagine $\gamma$-nitrogen in nucleotide biosynthesis in cancer cells hijacked by an oncogenic virus. MBio. 2017;8(4):e01179-e011717. doi:10.1128/mBio.01179-17

28. Chaiyawat P, Settakorn J, Sangsin A, et al. Exploring targeted therapy of osteosarcoma using proteomics data. Onco Targets Ther. 2017;10:565-577. doi:10.2147/OTT.S119993

29. Liu F, Ma F, Wang Y, et al. PKM2 methylation by CARM1 activates aerobic glycolysis to promote tumorigenesis. Nat Cell Biol. 2017;19 (11):1358-1370. doi:10.1038/ncb3630

30. Zhou Z, Li M, Zhang L, et al. Oncogenic kinase-induced PKM2 tyrosine 105 phosphorylation converts nononcogenic PKM2 to a tumor promoter and induces cancer stem-like cells. Cancer Res. 2018;78(9):2248-2261. doi:10.1158/0008-5472.CAN-17-2726

31. Ao R, Guan L, Wang Y, Wang JN. Effects of PKM2 gene silencing on the proliferation and apoptosis of colorectal cancer LS-147T and SW620 cells. Cell Physiol Biochem. 2017;42(5):1769-1778. doi:10.1159/000479456

32. Guo CY, Yan C, Luo L, et al. Enhanced expression of PKM2 associates with the biological properties of cancer stem cells from A549 human lung cancer cells. Oncol Rep. 2017;37(4):2161-2166. doi:10.3892/or.2017.5438

33. Zhao Z, Song Z, Liao Z, et al. PKM2 promotes stemness of breast cancer cell by through Wnt/ $\beta$-catenin pathway. Tumour Biol. 2016;37 (3):4223-4234. doi:10.1007/s13277-015-4121-8 
34. Wang X, Xu Y, Wang X, et al. LincRNA-p21 suppresses development of human prostate cancer through inhibition of PKM2. Cell Prolif. 2017;50(6). doi:10.1111/cpr.12368

35. Benesch C, Schneider C, Voelker HU, et al. The clinicopathological and prognostic relevance of pyruvate kinase $\mathrm{M} 2$ and pAkt expression in breast cancer. Anticancer Res. 2010;30(5):1689-1694.

36. Wong N, Ojo D, Yan J, Tang D. PKM2 contributes to cancer metabolism. Cancer Lett. 2015;356(2Pt A):184-191. doi:10.1016/j. canlet.2014.01.031

37. Yang W, Lu Z. Regulation and function of pyruvate kinase M2 in cancer. Cancer Lett. 2013;339(2):153-158. doi:10.1016/j. canlet.2013.06.008

38. Wang C, Delogu S, Ho C, et al. Inactivation of Spry2 accelerates AKT-driven hepatocarcinogenesis via activation of MAPK and PKM2 pathways. $J$ Hepatol. 2012;57(3):577-583. doi:10.1016/j. jhep.2012.04.026
39. Prigione A, Rohwer N, Hoffmann S, et al. HIF1 $\alpha$ modulates cell fate reprogramming through early glycolytic shift and upregulation of PDK1-3 and PKM2. Stem Cells. 2014;32(2):364-376. doi:10.1002/ stem. 1552

40. Yang W, Xia Y, Cao Y, et al. EGFR-induced and PKCe monoubiquitylation-dependent NF- $\kappa \mathrm{B}$ activation upregulates PKM2 expression and promotes tumorigenesis. Mol Cell. 2012;48(5):771-784. doi:10.1016/j.molcel.2012.09.028

41. Panasyuk G, Espeillac C, Chauvin C, et al. PPAR $\gamma$ contributes to PKM2 and HK2 expression in fatty liver. Nat Commun. 2012;3:672. doi: $10.1038 /$ ncomms 1667

42. Xu Q, Tu J, Dou C, et al. HSP90 promotes cell glycolysis, proliferation and inhibits apoptosis by regulating PKM2 abundance via Thr-328 phosphorylation in hepatocellular carcinoma. Mol Cancer. 2017;16(1):178. doi:10.1186/s12943-017-0748-y

\section{Publish your work in this journal}

OncoTargets and Therapy is an international, peer-reviewed, open access journal focusing on the pathological basis of all cancers, potential targets for therapy and treatment protocols employed to improve the management of cancer patients. The journal also focuses on the impact of management programs and new therapeutic agents and protocols on patient perspectives such as quality of life, adherence and satisfaction. The manuscript management system is completely online and includes a very quick and fair peer-review system, which is all easy to use. Visit http://www.dovepress.com/ testimonials.php to read real quotes from published authors. 